\title{
ESTIMATION OF INDUCTION MOTOR MODEL PARAMETERS - CASE STUDY
}

\author{
Mahmoud A. Al-Ahmar \\ Electrical Engineering Department, Faculty of Engineering, Benha University \\ Cairo, Egypt \\ E-mail: mahmoud.alahmar@feng.bu.edu.eg
}

\begin{abstract}
Modern control methods in the operation of different electrical machines are essential to develop an integrated mathematical model for the machine. The model is to calculate its inputs and outputs, which should match the performance of the machine. In order that, the model gives accurate inputs and outputs that matching the machine, it is necessary to introduce model parameters that are calculated according to machine rating as well as some laboratory measurements on the machine. In this research, a model of three phase induction motor is used to obtain the parameters of the proposed model using different methods. The calculations using that model can provide all laboratory operating results of the motor and satisfy its data sheet information. In this paper, different methods are used to estimate the parameters; curve fitting algorithm and iteration method. Accurate results of the model are obtained. The obtained results from the different methods are compared with the measured and show good agreement. Utilizing the slip-torque and slip-current motor characteristic, the estimation method is exhibited with some basic numerical illustrations.

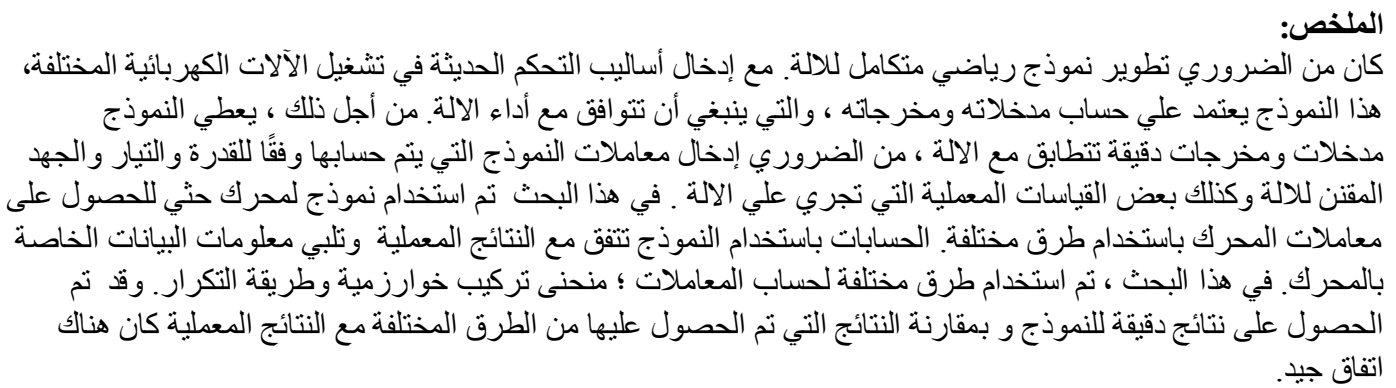

Keywords: Model of Induction motor; Curve fitting, Parameter estimation, System Identification.

\section{INTRODUCTION}

With the huge development in the components of electronics and using computers and microprocessors, it becomes necessary to use the modern methods in controlling different machines.

The main aim is to develop the characteristics of these machines, increasing its efficiency and satisfy speed and accurate performance. Therefore, mathematical models of the machines are developed and the parameters of these models are estimated. This is to enable in calculating the performance of the machines in different running conditions. And with feedback information to the model in case of any change to the machine outputs, so as to correct the variables of the machine quickly to keep its characteristics in the optimal state, in spite of different running conditions. With the last development in electronic components, different systems arise can be used to achieve big development in the performance of the electrical motors. In this research, the three phase induction motor is used as a case study. With these motors, two modern systems are used for running; these are:

1- Direct torque control

2- Field oriented control

Using these systems, the motor's torque increases with high percentage during the starting period and when changing the motor speed. Also the electrical currents of the motor are reduced considerably with the same loads. The motor efficiency is increased to reach the maximum efficiency in spite of changing the load of the motor. These developments occur 
quickly as soon as load change. Hence, the system will be with a rapid response. To achieve these advantages, the mathematical model of the motor must depend on the motor's parameters. This is necessary, because when fixed or constant parameters were used, the system will run efficiently in certain running conditions. These certain conditions may change as a result of different reasons. First, when the speed varied as a result of load variation. Secondly; if the frequency of the voltage on the motor changes. In reference [1] a proposes technique, supported the differential evolution rule, geared toward the estimation of the electrical and mechanical parameters of three-phase induction motors. Such rule was capable of estimating the parameters of the equivalent electrical circuit, corresponding to stator and rotor resistances and leakage inductances, the magnetizing inductance, and conjointly mechanical parameters, corresponding to moment of inertia and therefore the friction constant.

With the new running conditions of the machine, the parameters of the motor will change naturally. To maintain running the system efficiently, the actual values of the parameters should be introduced. Otherwise, all the rotor characteristics will deviate from the ordinary running conditions. The resistance of the rotor $\left(\mathrm{R}_{2}\right)$ is the most parameter changing with changing running conditions; especially with rotors of the deep bar type or double cage. The changeable second parameter is the reactance of the rotor $\left(\mathrm{X}_{2}\right)$.

The parameters of a given machine is identified by [2]. The used method is based on data assimilation and the finite element method. A particle swarm optimization method-based multi-objective parameter estimation of three different induction motor models has been projected in reference [3]. A new algorithm using the immune algorithm (IA) to optimize the parameters of three different induction motor models from the manufacturer data and/or from the tests was conferred by [4]. An improved slippy mode observer with the parameter error compensation for the speed sensorless control of an induction motor was investigated by [5]. The proposed parameter estimation is obtained from the error between the measured and decoupled currents. In power system load modeling is of great importance, for both steady-state and transient analysis. It has been tended to broadly in literatures [6] - [9]. Induction motors constitute the most critical part of modern load and their execution considerably affects the behavior of power system. One of the primary issues with induction representation is the inaccessibility of parameter qualities to build precise models.
This is one in every of the explanations that induction motors are not sometimes diagrammatic in system studies. Several investigations of induction motor parameter estimation has been addressed [10][15]. In many cases high accuracy is required in the parameter estimation, when the problem is viewed from the electric machines point of view [10]-[13]. However, for power system analysis when load modeling is concerned the level of accuracy needed is considerably less, of the order of $(10-15) \%$ [14]. Field measurements after particular tests can be utilize for precise motor model estimation, however such test are normally difficult to perform in real, operational industrial motors [15]. Besides, performing such tests have neither rhyme nor reason when the precision necessities are moderately low.

Therefore, many approaches are conferred for induction model identification supported information that may become simply obtainable [14]-[15]. Just about every last one of methodologies plan those estimation issue likewise a nonlinear minimum squares minimization issue that estimates every last one of obscure parameters at the same time.. This problem can be solved either numerically (Newton type methods) [15] or by intelligence-based techniques (genetic algorithms) [14]-[15].

Numerical systems would thick, as efficient; however, they are accounted not to be exact strong and inclined will give suboptimal results alternately no result at all, relying upon those firmness of the issue also on the introduction of the iterative result calculation [14]-[15]. Furthermore, they oblige beneficial information of the explanatory models utilized [14] - [15]. Due to these disadvantages, intelligence techniques have been used that require little data on the proposed model and are more robust, at the expense of large amount of computational time [14]-[15] and lack of insight in the problem solution.

This paper concentrates on the implementation of an induction motor parameter calculation algorithm that is based on numerical methodology. The planned methodology is applied to the induction motor models and estimates the model rotor parameters using the torque-slip characteristic. For the estimation of all the motor equivalent circuit parameters the torque-slip characteristic alone is not enough and conjointly the current-slip characteristic or the power factor-slip characteristic may be used, to produce additional information. Such characteristics may be most of the times obtained by the manufacturers. Here the characteristics are assumed to be known as a number of discrete points. 


\section{PERFORMANCE ANALYSIS OF THE \\ MACHINE}

Induction machine model has been developed that can more accurately describe the machine behavior. The characteristics of the machine are obtained from the equations that obtained from the equivalent circuit shown in Fig. 1.

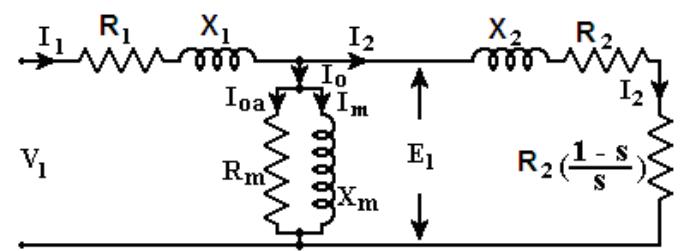

Fig. 1. Machine equivalent circuit.

where;

$\mathrm{R}_{1}=$ stator phase resistance

$\mathrm{R}_{2}=$ rotor phase resistance referred to stator.

$\mathrm{R}_{\mathrm{m}}=$ iron loss phase resistance.

$\mathrm{X}_{1}=$ stator phase leakage reactance.

$\mathrm{X}_{2}=$ rotor phase leakage reactance at standstill referred to stator.

$\mathrm{X}_{\mathrm{m}}=$ phase magnetizing reactance.

$\mathrm{I}_{1}=$ stator phase current.

$\mathrm{I}_{2}=$ rotor phase current referred to stator.

$\mathrm{I}_{0}=$ no load phase current.

$\mathrm{I}_{0 \mathrm{a}}=$ equivalent iron loss phase current.

$\mathrm{I}_{\mathrm{m}}=$ phase magnetizing current.

$\mathrm{E}_{1}=$ stator phase e.m.f.

$\mathrm{E}_{2}=$ rotor phase e.m.f.

$\mathrm{E}_{20}=$ rotor phase e.m.f. at standstill referred to stator.

$\mathrm{V}_{1}=$ input phase voltage.

$\mathrm{S}=$ slip.

The proposed model described is based on the equivalent circuit of an induction motor. The equation of equivalent impedance $Z_{T}$ is calculated by knowing the equivalent circuit parameters at any value of the slip from equation ( 1 ):

$$
Z_{T}=\mathrm{R}_{\mathrm{T}}+j X_{T}
$$

Where:

$$
\begin{aligned}
& R_{T}=R_{21}+R_{1} \\
& X_{T}=X_{21}+X_{1}
\end{aligned}
$$

$$
\begin{aligned}
& R_{21}=\left(R_{M M} R_{2 M}+X_{M M} X_{2 M}\right) / A_{Z 2} \\
& X_{21}=\left(X_{M M} R_{2 M}-R_{M M} X_{2 M}\right) / A_{Z 2} \\
& A_{Z 2}=\left(R_{2 M}^{2}+X_{2 M}^{2}\right) \\
& R_{M M}=\left(R_{2 S} R_{Z M}-X_{2} X_{Z M}\right) \\
& X_{M M}=\left(X_{2} R_{Z M}+R_{2 S} X_{Z M}\right) \\
& R_{2 M}=R_{2 S}+R_{Z M} \\
& X_{2 M}=X_{2 S}+X_{Z M} \\
& R_{2 S}=R_{2} / s \\
& X_{2 S}=X_{2} \\
& A_{Z M}=\left(R_{M}^{2}+X_{M}^{2}\right) \\
& R_{Z M}=R_{M} X_{M}^{2} / A_{Z M} \\
& X_{Z M}=R_{M}^{2} X_{M} / A_{Z M}
\end{aligned}
$$

Then, the phase current of the stator is calculated by:

$I_{1}=V / Z_{T}$

Also, the power factor is calculated as:

Pf $=R_{T} / Z_{T}$

And the input power of the motor is calculated by:

$\mathrm{P}_{1}=3 \mathrm{VI}_{1} \mathrm{pf}$

The rotor current referred to stator is calculated as:

$I_{2}=E / Z_{2 S}$

Where,

$$
Z_{2 S}=\sqrt{R_{2 S}^{2}+X_{2 S}^{2}}
$$

Therefore, the output power of the motor can be obtained

from the equation:

$p_{2}=3 I_{2}^{2} R_{2}(1-s) / s$

And the torque is calculated from equation (21) as:

$T=9.554 P_{2} / N$

The iron losses $p_{i}$ of the motor is obtained as :

$p_{i}=3 E^{2} / R_{M}$

Where,

$E=I_{1} \cdot Z_{21}$

And, 
$z_{21}=\sqrt{R_{21}^{2}+X_{21}^{2}}$

The cupper losses $p_{c}$ are calculated from equation (25) as:

$p_{c}=3\left(I_{1}^{2} R_{1}+I_{2}^{2} R_{2}\right)$

From the supply frequency (f), the number of motor poles (2p), the supply voltage $(\mathrm{V})$, and the motor parameters $\left(\mathrm{R}_{1}, \mathrm{X}_{1}, \mathrm{R}_{2}, \mathrm{X}_{2}, \mathrm{R}_{\mathrm{M}}, \mathrm{X}_{\mathrm{M}}\right)$, the motor characteristics are computed from the previous equations.

The motor characteristics at any speed $\mathrm{N}$, is computed by the calculated slip $\mathrm{S}$ from the equation:

$\mathrm{S}=\left(\mathrm{N}_{\mathrm{S}}-\mathrm{N}\right) / \mathrm{N}_{\mathrm{S}}$

Where, $\mathrm{N}_{\mathrm{S}}$ is the synchronous speed.

Using the rest of motor parameters, the performance characteristics are calculated, especially the torque / speed relation.

In this work, for accurate computation, a generalized model is proposed that assumes the rotor parameters are not constant through the variation of speed. A polynomial dependence of the forth order is used for the rotor resistance and the rotor reactance.

\section{MOTOR PARAMETERS ESIMATION}

\section{(a) Problem Formulation}

The estimation approach makes utilization of information that, as a rule, can get to be distinctly accessible from the motor manufacturer data, or are easily measured. Like the torque / speed characteristics, or the current / speed or power factor / speed characteristics. The parameters estimation procedure becomes additional necessary, once a slipdependent parameter model is employed. During this case, the model coefficients of equations (1) to (26) are difficult to be obtained while not the employment of associate estimation procedure.

Once the rotor parameters are to be a calculable, assumptive that the stator and the core parameters are given, the torque / speed characteristics of the motor, provides enough information. However, once all the equivalent circuit parameters are to be calculable the torque / speed characteristics itself are not enough. Some additional information's are necessary.

Such information can be provided using, for instance, the current / speed characteristic of the motor along with the torque / speed curve. During this paper the estimation problem are outlined in its general form, assuming that all the equivalent circuit parameters are constant and the performance of the machine are calculated at different speeds. The obtained torque / speed relation is shown in Fig .2.

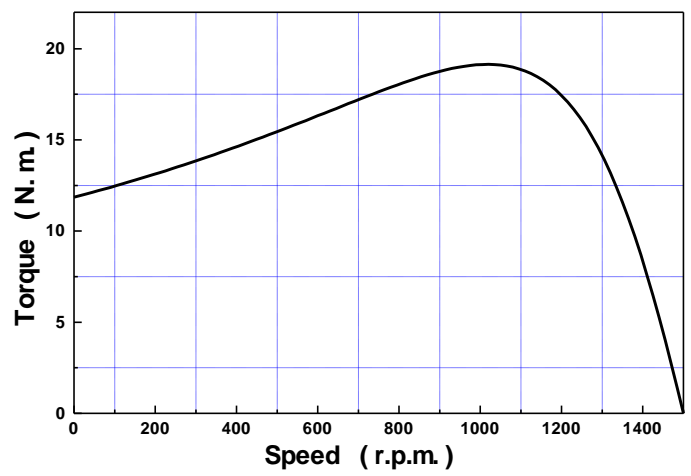

Fig. 2. Torque / speed Characteristics at constant parameters.

This curve gives the same data sheet full load torque at full load speed of 1400 r.p.m. But the data sheet starting torque at zero speed is higher than that given in this figure. Therefore, the objective of this paper is to obtain a relation of varying $R_{2}$ and $X_{2}$ with the speed. Since the rotor resistance and reactance are the most parameters alterable with the speed due to skin effect.

(b) Curve Fitting of $\mathbf{R}_{2}$

In this first method, "Curve Fitting of $\mathrm{R}_{2}$ ", the principal equations of the motor are used; equations (1) - (22) in calculating the torque of the motor starting from standstill to reach the synchronous speed. At each speed, the value of the rotor resistance $\mathrm{R}_{2}$ is assumed and decreased according to an iterative computing method to obtain the value of $R_{2}$ that gives the torque value that obtained in the data sheet of the motor. Thus, different values of $R_{2}$ are obtained at the different speeds. These values of $R_{2}$ are shown in Fig. 3.

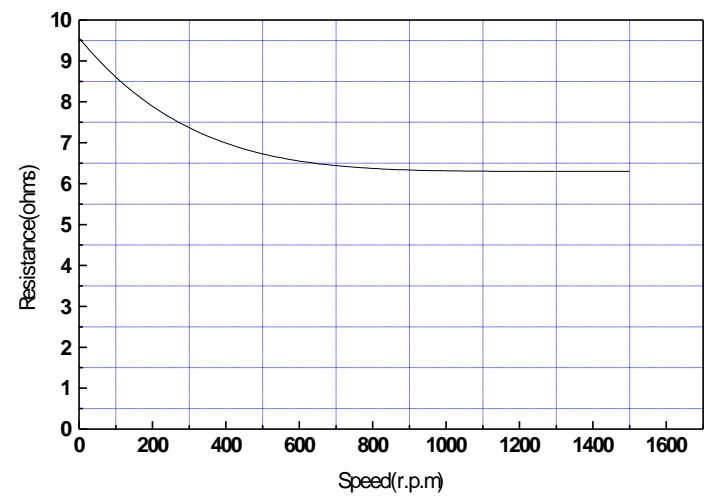

Fig. 3.Variation of rotor resistance with speed.

From that curve of $\mathrm{R}_{2}$, a fitting formula is obtained using a curve fitting technique. This formula is given by : 
$\mathrm{R}_{2}=9.5483128-0.10615459 \mathrm{~N}+1.3293197 * 10^{\wedge}(-$ 5) $\mathrm{N}^{\wedge} 2-7.5179658 * 10^{\wedge}(-9) \mathrm{N}^{\wedge} 3+1.6098777 * 10^{\wedge}(-$ 12) $\mathrm{N}^{\wedge} 4$

Using this formula given by (28), in a program computing the performance characteristics of the machine, the torque / speed relationship is obtained as shown in Fig. 4.

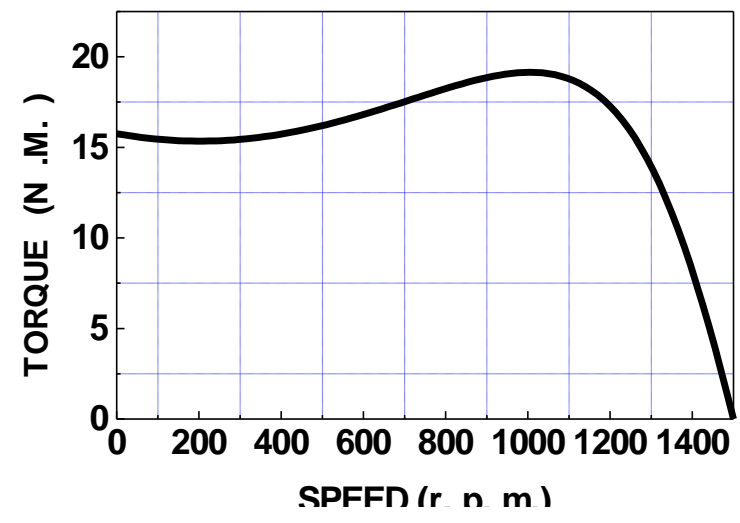

Fig. 4. Torque / speed relationship using $\mathrm{R}_{2}$ curve fitting

In this figure, the starting torque and full load torque are equal to that given in the data sheet. But the maximum torque is still higher than that of the data sheet. This is due to the constant value of rotor reactance $\mathrm{X}_{2}$. Curve fitting of $\mathrm{X}_{2}$ is necessary to correct the value of $\mathrm{X}_{2}$

\section{( C ) Curve Fitting of $\mathbf{X}_{2}$}

Also, in this method, as with the case of $R_{2}$, the principal equations of the motor are used in calculating the torque of the motor starting from standstill until reaching the synchronous speed. At each speed, the value of the rotor reactance $X_{2}$ is assumed and increased according to an iterative method to obtain the values of $\mathrm{X}_{2}$ that gives the torque value obtained in the data sheet of the motor. So different values of $X_{2}$ are obtained at each speed as shown in Fig. 5.

The curve fitting technique is applied on the reactance /speed relationship shown in Fig. 5. When keeping the rotor resistance at constant value, the obtained formula of the rotor reactance as a function of speed is given as follows :

$\mathrm{X}_{2}=8.7799992+0.009760012 \mathrm{~N}-3.25332693$

$* 10^{\wedge}(-6) \mathrm{N}^{\wedge} 2+3.7722738 * 10^{\wedge}(-14) \mathrm{N}^{\wedge} 3$

$+1.2512177 * 10^{\wedge}(-17) \mathrm{N}^{\wedge} 4$

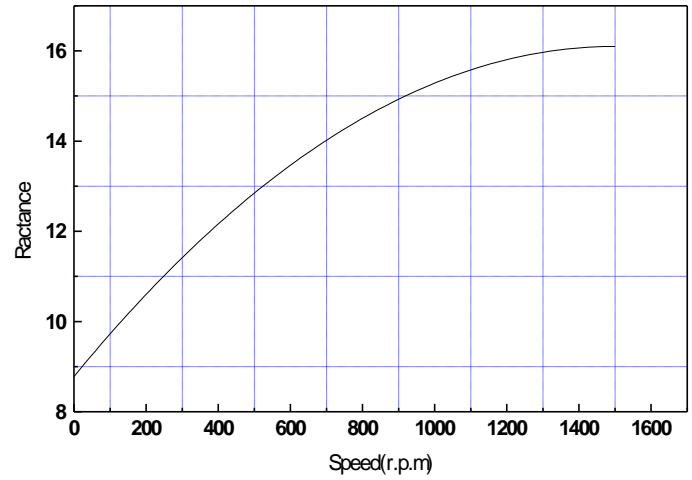

Fig. 5 Variation of rotor reactance with speed.

The Torque / Speed curve obtained with curve fitting for both R2 and X2 is given in Fig. 6. All values of starting torque, maximum torque and full load torque of this curve are equal to those of the motor data sheet.

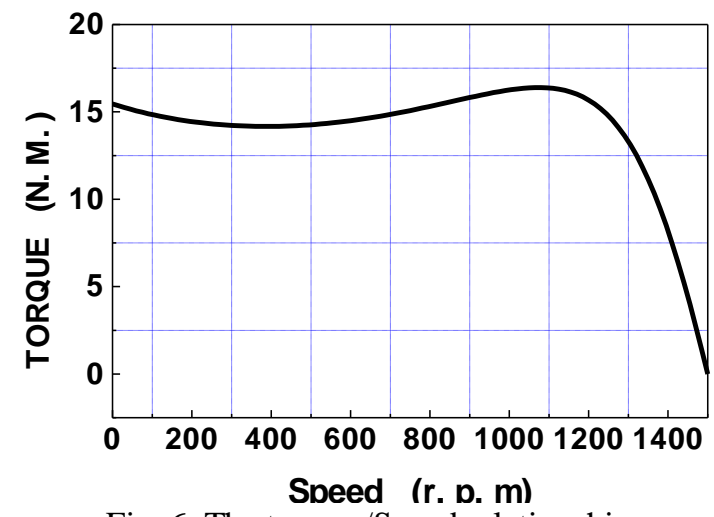

Fig. 6. The torque/Speed relationship.

A comparison of various cases is shown in Figs. 7 and 8 for torque / speed and current / speed relationships respectively.

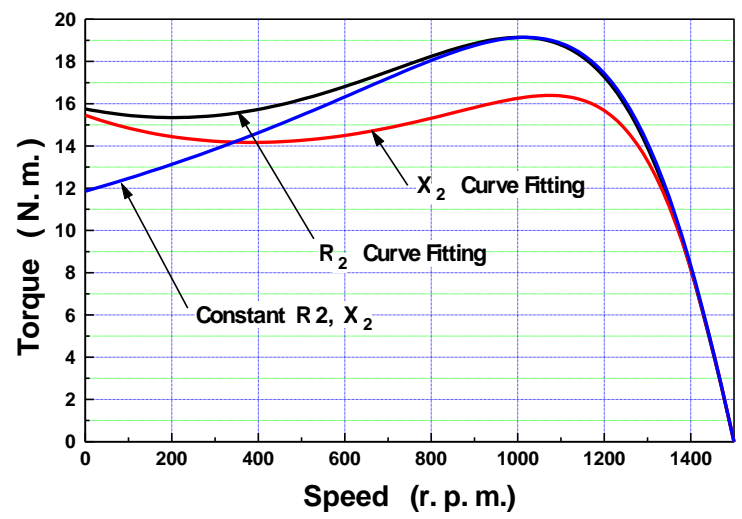

Fig. 7. Torque / Speed relationship for different approaches 


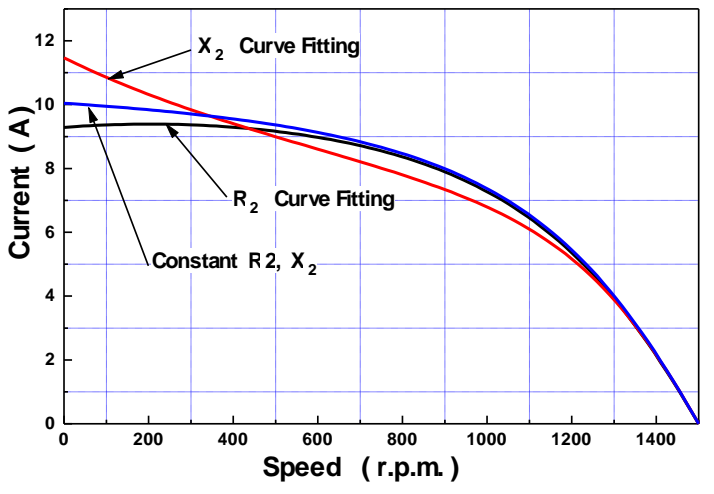

Fig. 8. Current / speed relationship for different approaches.

\section{CONCULSION}

This paper presents a model parameters estimation methodology. The model permits the identification of a machine model. Especially when these parameters are varied with operation. The methodology is based on simulation of curve fitting. Comparative study between three techniques of parameters estimation is obtained. These are; firstly by a constant parameters for the machine. Secondly, a curve fitting technique for the rotor resistance estimation is used. Finally, the curve fitting technique is used for rotor reactance estimation. A comparative study of various cases is presented. The torque-speed and current-speed relationships are shown for different approaches. A comprehensive study of the mentioned methodology shows that the curve fitting strategy of rotor reactance gives more accurate results than other approaches.

\section{REFRENCES}

[1] Jacqeline Jordan Guedes, Marcelo Favoretto Castoldi, Alessandro Goedtel,,Cristiano Marcos Agulhari and Danilo Sipoli Sanches,

"Parameters estimation of three-phase induction motors using differential evolution", Electric Power Systems Research, Vol ,154, PP. 204-212, 2018.

[2] A. Bacchus, A. Toonzi, G. P. Argaud, M. Biet, L. Mocaire and Y. Le Menach,"Estimation of FEM parameters using data assimilation and its application to an electrical machines", IEEE Transactions onMagnetics, Vol.52,No.3,March 2016.

[3] Sakthivel, V. P., Bhuvaneswari, R. and Subramanian, S. " Multi-objective parameter estimation of induction motor using particle swarm optimization," Engineering

Applications 2010. of Artificial Intelligence Vol. 23, No.3,pp.302-312.

[4] Sakthivel, V. P., Bhuvaneswari, R. and Subramanian, S. " Artificial immune system for parameter estimation of induction motor," Expert Systems with Applications, Vol .37, No.8, pp. 6109-6115, 2010.

[5] Yong Hun Park, Kang Hyo Park, Seung Chul

Baek, and Young Ahn kwon "Sliding mode observer with parameter estimation for sensorless induction motor," IEEE Conference (TENCON2010)-Fukuoka Region 10, pp.37-41, 2010.

[6] K. Morison, H. Hamadani, and L. Wang, "Practical issues in load modeling for voltage stability studies," in Proc. 2003 IEEE-PES General Meeting, Toronto, Canada, July 13-17, 2003.

[7] K. Tomiyama, S. Ueoka, T. Takano, I. Iyoda, K. Matsuno, K. Temma, and J. J.Paserba,"Modeling of Load During and After System Faults Based on Actual Field Data," in Proc. 2003, IEEE-PES General Meeting, Toronto, Canada, July 13-17, 2003.

[8] F. P. de Mello, and J. W. Feltes, "Voltage oscillatory instability caused by induction motor loads," IEEE Trans. on Power Systems, Vol. 11, No. 3, pp. 1279-1285,Aug. 1996.

[9] B. R. Williams, W. R. Schmus and D. C. Dawson, Transmission voltage recovery delayed by stalled air conditioner compressors," IEEE Trans. on Power Systems, Vol. 7, No. 3, pp. 1173-1181, Aug.1992.

[10] Z. Zhang, G. E. Dawson, and T. R. Eastham, "Evaluation of dynamic parameters and performance of deep-bar induction machines," in Proc. IEEE-IAS Annu. Meeting, pp. 62-66, 1993.

[11] S. I. Moon and A. Keyhani, "Estimation of induction machine parameters from standstill time domain data," in Proc. IEEE-IAS Annu. Meeting, pp.336-342, 1993.Energy Conversion, Vol. 4, pp. 608-615, Dec. 1989.

[12] L. Zai, C. L. de Marco, and T. A. Lipo, "An extended Kalman filter approach to rotor time constant measurement in PWM induction motor drives," IEEE Trans. Ind. Applications, Vol. 28, pp. 96-104, Jan. /Feb. 1992.

[13] J. A. de Koch, F. S. van der Merwe, and H. J. Vermeuler, "Induction motor parameter estimation through and output error technique," presented at the IEEE-PES Winter Meeting, Columbus, OH, 1993, Paper 93-WM 01909 EC.

[14] P. Pillay, R. Nolan, and T. Haque, "Application of genetic algorithm to motor parameter determination for transient torque calculations," IEEE Trans. Ind. Applications, Vol. 33,No 5, pp. 1273-1282, Sept. /Oct.1997.

[15] T. Phumiphak and C. Chatuthai, "Estimation of induction motor parameters based on field test coupled with genetic algorithm," in Proc. Int. Conf. Power System Technology, pp. 1199-1203, 2002 (Power Con 2002). 